\title{
SOLAR MICROWAVE EMISSION ASSOCIATED WITH CORONAL MASS EJECTIONS (CME)
}

\author{
J. Kallunki \\ Aalto University Metsähovi Radio Observatory, \\ Metsähovintie 114, Kylmälä, 02450, FINLAND \\ e-mail: juha.kallunki@aalto.fi
}

The connection between Coronal Mass Ejections (CME) and radio burst has been discovered especially at lower frequencies $(<2 \mathrm{GHz})$. The aim of the study is to investigate possible connection between CMEs and variability of radio brightenings at $37 \mathrm{GHz}(8 \mathrm{~mm})$ within the time frame of four days. The millimetre radio observations have been made on RT-14 radio telescope at Metsähovi Radio Observatory of Aalto University, Finland. In addition, $11.2 \mathrm{GHz}$ $(2.7 \mathrm{~cm})$ total solar flux information is included in the analysis. The radio observations were made between March 2011 and September 2017, totally including 24 events. The results demonstrate that in most of the cases the radio brightening intensity achieves its maximum before CME occurs. Time of $11.2 \mathrm{GHz}$ intensity appearance matches with time of CME appearance with difference of two to three hours. However, in most cases a maximum of $11.2 \mathrm{GHz}$ intensity appears before CMEs. The study investigates a possibility of predicting CME appearance based on milli- and centimetre radio observations. The study also proposes a scenario connection between CMEs and solar microwave events.

Keywords: Coronal Mass Ejection (CME), radio telescope, solar activity, solar radio brightening. 
Coronal Mass Ejections (CMEs) are strong eruptive events which are carrying magnetized plasma from the solar atmosphere. CMEs, for instance, are causing geomagnetic storms. Thus, they play a major role in the space weather investigations. They can carry massive kinetic energies, up to $10^{34} \mathrm{erg}$ [1]. The formation of CMEs is not yet comprehensive understood. However, with the current knowledge, they are assumed to be driven by magnetic fields [2]. Also, there have been discussions about the connection between $\mathrm{CME}$ and solar flare.

The estimation of CME magnetic fields can be diagnostic based on radio observations [3], [4]. Connection between CMEs and solar radio events has been studied earlier. However, most of the studies have been focusing on lower frequencies $(<2.5$ $\mathrm{GHz}$, e.g., [1]-[5]). For instance, connection between solar type II radio burst and CME has been studied in a versatile manner [5].

Type II solar radio bursts are generated by the plasma emission mechanism when electron beams are accelerated ahead of propagating CME [6]. At millimetre wavelength regimes, a comparison to X-ray events has also been investigated [7]. The results show that a gradual thermal component of microwave starts to dominate after the impulsive phase of X-ray flare. CMEs have not been studied at higher radio frequencies. The present article studies the evolution of the radio brightenings at $37 \mathrm{GHz}$ which are associated with CME. The main aim is to find possible connection between CMEs and radio brightenings. For instance, CMEs can be predicted based on radio brightening evolution. The] most common behaviour seems to be that the radio brightening intensity achieves its maximum before the CME occurs. Also, in most cases $11.2 \mathrm{GHz}$ solar radio flux maximum intensity is achieved before CME appears. In Section 2, the used radio instrumentation is shown. Observations are discussed in Section 3 and results - in Section 4. Finally, Section 5 presents conclusions.

\section{INSTRUMENTATION}

The RT-14 at Metsähovi Radio Observatory (MRO) of Aalto University, Finland (Helsinki region; E 24:23.35, N 60:13.04) is a radome-enclosed Cassegrain-type antenna with a diameter of $13.7 \mathrm{~m}$. The usable wavelength range of the telescope is 13.0 $\mathrm{cm}-2.0 \mathrm{~mm}$. During solar observations, the antenna is used for solar mapping, partial solar mapping, and tracking of any selected areas on the solar disk. The beam size of the telescope is $2.4 \mathrm{arc} \mathrm{min}$ at $37 \mathrm{GHz}(8 \mathrm{~mm})$. The receiver is Dicke-type radiometers. For the temperature stabilization, a Peltier element is used. The noise temperature of the $8 \mathrm{~mm}$ receiver is approximately $280 \mathrm{~K}$. The temporal resolution during observations is $0.1 \mathrm{~s}$ or less. It will take approximately 180 seconds to make one solar radio map.

The observational data are recorded in intensities. Because the measurements are always scaled relative to the quiet Sun level (QSL), the observations are comparable over the years. The brightness temperature of the QSL at $8 \mathrm{~mm}$ is $8100 \pm \mathbf{3 0 0} \mathrm{K}$ [8]. 
The full documentation of the RT-14 technical specifications can be found in [9].

The second instrument is a radio telescope with a $1.8 \mathrm{~m}$ dish diameter (RT-1.8) dedicated to continuous solar observations. RT-1.8 has measured the total radiation of the Sun since January 2001, at a frequency of $11.2 \mathrm{GHz}(2.7 \mathrm{~cm})$. It has been in use for two solar cycles, 23 and 24. The long time series enables solar cyclicity studies. The telescope has a beam size of 81.6 arc min and its system noise temperature, $T_{s y s}$, is $268 \mathrm{~K}$, measured against liquid nitrogen. The radio telescope has no protective radome; therefore, it is vulnerable to prevailing weather conditions. High sampling rate $(1 \mathrm{kHz})$ allows studying fine structure of flares, including short periodic oscillation phenomena. The full documentation of the RT-1.8 technical specifications can be found in [10].

\section{OBSERVATION}

The radio observations were made between March 2011 and September 2017 and daily maximum brightness temperature value was recorded from daily observed solar raster scan maps. The radio brightenings were associated to NOAA (National Oceanic and Atmospheric Administration) active region, and connection between the active regions and CMEs was found. $\mathrm{CME}$ appearance times were collected from HELCATS - The science of tracking solar storms' database (https://www.helcats-fp7. eu). The CME appearance time is defined so when CME is first time observed in HI1 camera (wide-field white-light camera). The maximum radio brightening temperature values were taken during the period of four days, starting two days before CME occurred and stopping one day after CME occurred. Figure 1 presents the evolution of radio brightening between 4 September 2017 and 7 September 2017.

In the third plot (in lower left panel, radio map from 6 September 2017), we can notice that a radio brightening area is expanding compared to other days. In addition, the intensity is stronger. CME appeared on 6 September 2017.

Number of daily solar radio maps varied widely. On certain days, we only had one to three radio maps, but on some days we had more than two hundred radio maps. On the days with a dense sampling, we recorded the radio brightening intensity simultaneously when CME appeared. However, on days with a sparse sampling, we had rough estimate on its intensity. Thus, we probably missed the radio brightening intensity when CME appeared.

Totally, we found 24 events (Table 1), where we had both radio brightening and CME observations. In some cases, there were several CMEs during this period of four days. CMEs under investigation were chosen that the CME observation was classified into class "good". It means that eruption is defined with a good confidence as CME. The selection of the events was made based on radio brightenings. Even though there were more CME events in the period under review, we were missing the radio data on those events. Ground-based radio data are also certainly available only from the sunrise to the sunset. MRO-14 radio telescope is also used for other purposes than solar observations. Thus, we have only one solar radio map for each day. 
Table 1. CMEs and Radio Brightness Maxima (11 and $37 \mathrm{GHz}$ ) Occurrence Time (including radio brightness maximum intensities relative to the QSL)

\begin{tabular}{|c|c|c|c|c|}
\hline $\begin{array}{l}\mathrm{CME} \\
\text { occurrence time }\end{array}$ & $\begin{array}{l}37 \mathrm{GHz} \text { max. } \\
\text { intensity } \\
\text { occurrence time }\end{array}$ & $\begin{array}{l}37 \mathrm{GHz} \text { max. } \\
\text { intensity } \\
\text { (rel. to QSL) }\end{array}$ & $\begin{array}{l}11 \mathrm{GHz} \max . \\
\text { intensity } \\
\text { occurrence time }\end{array}$ & $\begin{array}{l}11 \mathrm{GHz} \text { max. } \\
\text { intensity } \\
\text { (rel. to QSL) }\end{array}$ \\
\hline 2011-03-07-14:48 & 2011-03-07 10:19 & 105.1 & 2011-03-07 09:20 & 149.1 \\
\hline 2011-03-14 16:09 & 2011-03-14 09:44 & 104.4 & No detection & No detection \\
\hline 2011-04-17 14:09 & 2011-04-17 12:16 & 111.1 & No detection & No detection \\
\hline 2011-06-02 10:49 & 2011-06-02 14:11 & 106.9 & No detection & No detection \\
\hline 2011-06-07 06:49 & 2011-06-07 04:19 & 105.6 & 2011-06-07 06:25 & 181.8 \\
\hline $2011-06-23$ 19:29 & $2011-06-23$ 03:57 & 105.4 & No detection & No detection \\
\hline 2011-07-09 04:09 & 2011-07-09 10:34 & 104.3 & No detection & No detection \\
\hline 2011-08-02 10:49 & 2011-08-02 03:52 & 112.3 & 2011-08-02 06:10 & 121.8 \\
\hline $2011-08-30 \quad 15: 29$ & 2011-08-03 04:00 & 137.9 & $2011-08-03$ 13:30 & 129.1 \\
\hline 2011-08-04 05:29 & 2011-08-04 03:42 & 124.2 & 2011-08-04 03:55 & 192.3 \\
\hline 2011-08-09 09:29 & 2011-08-09 10:52 & 105.2 & 2011-08-09 08:05 & 192.3 \\
\hline 2012-05-16 10:09 & 2012-05-16 04:07 & 105.1 & No detection & No detection \\
\hline 2012-05-17 06:09 & 2012-05-17 04:00 & 106.5 & No detection & No detection \\
\hline $2012-06-13$ 15:29 & 2012-06-13 04:08 & 109.8 & $2012-06-13$ 13:35 & 110.9 \\
\hline 2013-04-11 09:29 & 2013-04-11 11:05 & 111.9 & 2013-04-11 07:10 & 129.6 \\
\hline 2013-05-01 06:09 & 2013-05-01 04:36 & 110.4 & 2013-05-01 14:10 & 103.7 \\
\hline $2013-05-17$ 10:49 & 2013-05-17 09:32 & 140.2 & 2013-05-17 08:55 & 181.8 \\
\hline 2013-06-05 05:29 & 2013-06-05 05:01 & 103.8 & 2013-06-05 08:35 & 124.5 \\
\hline $2013-08-06 \quad 07: 29$ & 2013-08-06 04:11 & 105.0 & No detection & No detection \\
\hline 2013-08-17 12:09 & 2013-08-17 06:11 & 105.5 & No detection & No detection \\
\hline 2014-09-03 06:09 & 2014-09-03 04:33 & 106.7 & 2014-09-03 13:35 & 131.9 \\
\hline $2016-07-17$ 14:49 & 2016-07-17 08:21 & 114.6 & No detection & No detection \\
\hline 2017-05-06 08:49 & 2017-05-06 09:37 & 102.7 & No detection & No detection \\
\hline 2017-09-06 14:09 & 2017-09-06 09:38 & 198.4 & 2017-09-06 12:00 & 222.2 \\
\hline
\end{tabular}

In addition, $11.2 \mathrm{GHz}$ solar intensity was considered. CME occurrence time and $11.2 \mathrm{GHz}$ solar intensity maximum occurrence time had not longer than three hours of cadence. Figure 2 presents 11.2 GHz solar total flux on 6 September 2017.
There were three radio bursts before CME appeared. CME appeared on a gradual state of the third burst. Figures 3 and 4 show other examples. These observations were made between 31 July and 3 August 2011. During this period, several CMEs appeared. 


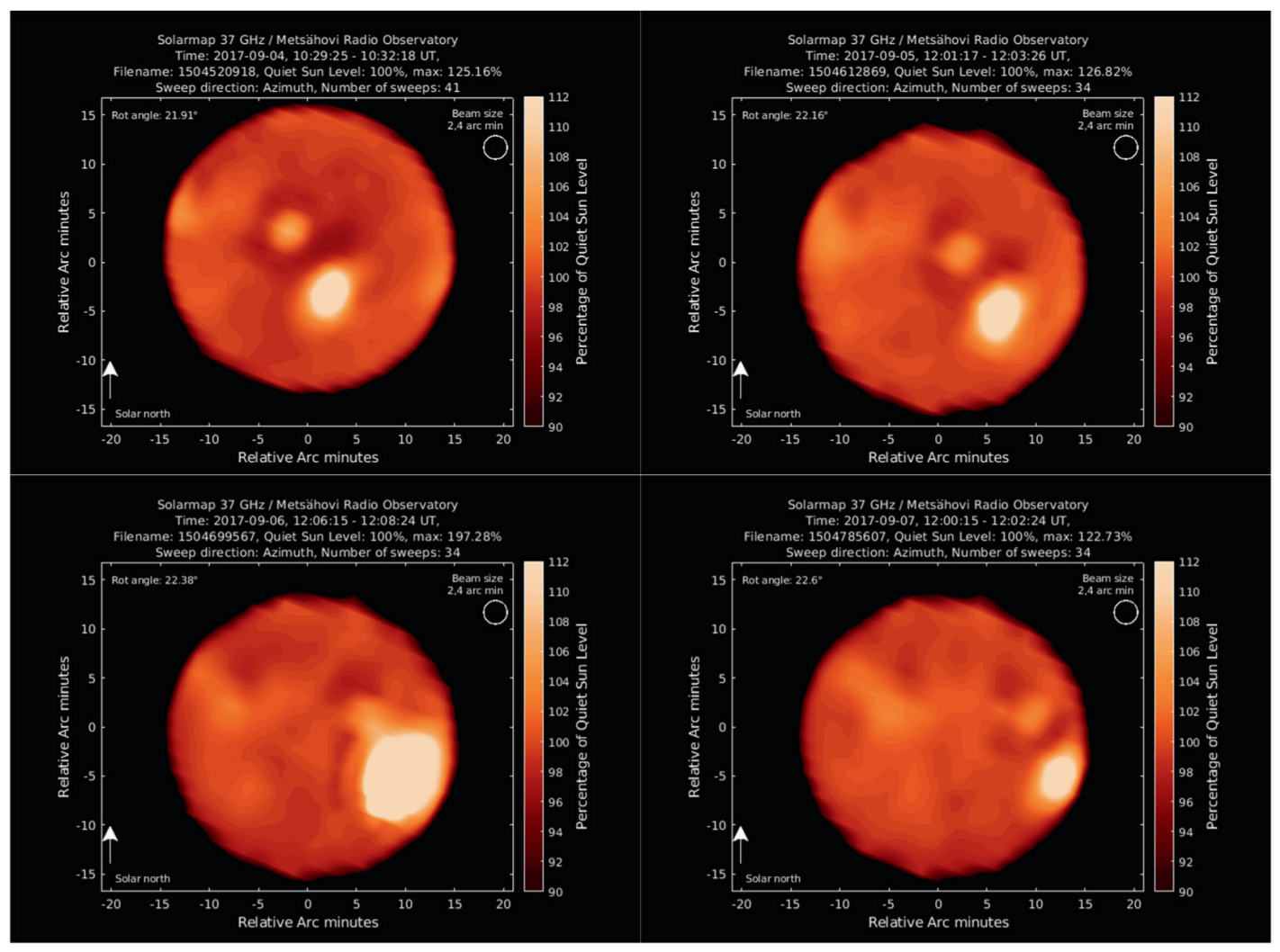

Fig. 1. Evolution of radio brightening between 4 September 2017 (upper left panel) and 7 September 2017 (lower right panel). Radio brightening size and intensity are on top on 6 September 2017 (lower left panel).

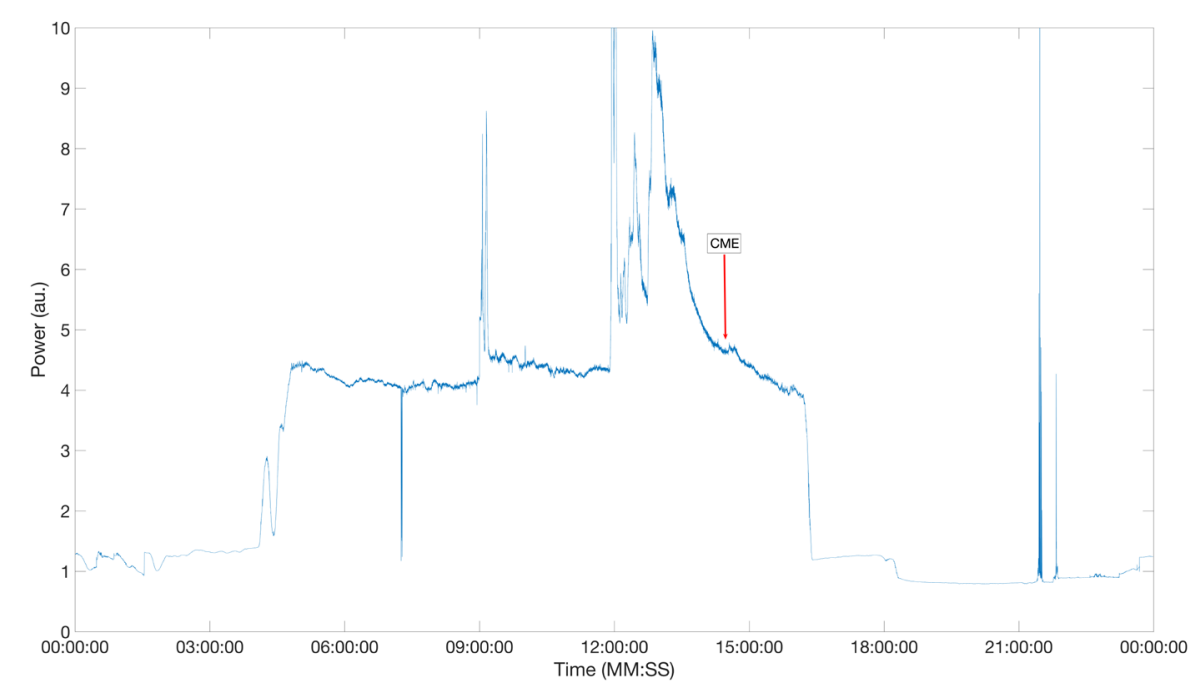

Fig. 2. The solar total intensity at $11.2 \mathrm{GHz}$ on 6 September 2017. CME appearrence time is indicated with an arrow. 

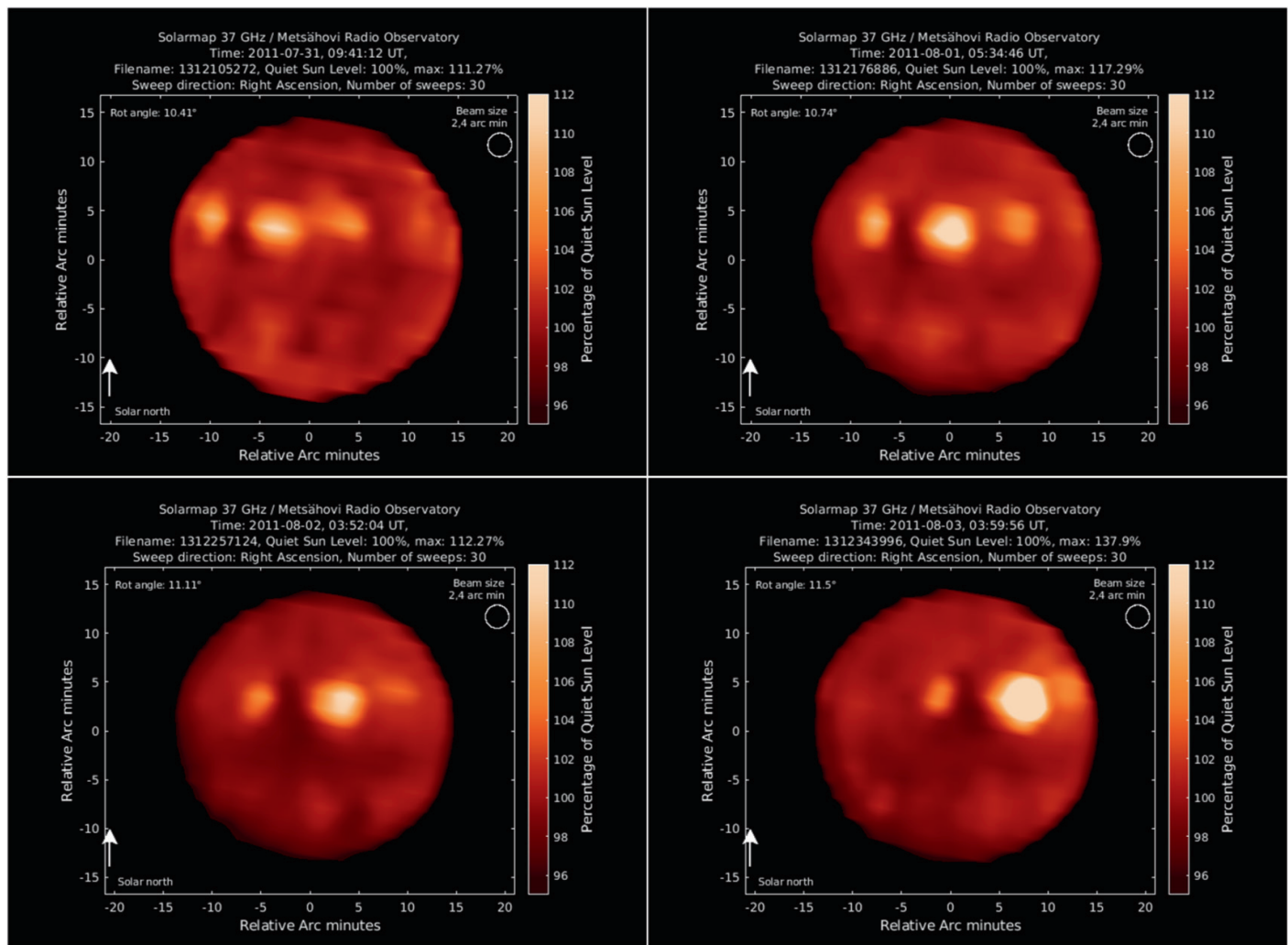

Fig. 3. Evolution of radio brightening between 31 July 2011 (upper left panel) and 3 August 2011 (lower right panel). Radio brightening size and intensity are on top on 3 August 2011 (lower right panel), one day after the CME appeared. This is explained by the fact that there was another CME on 3 August 2011. A weak solar burst appeared after CME.

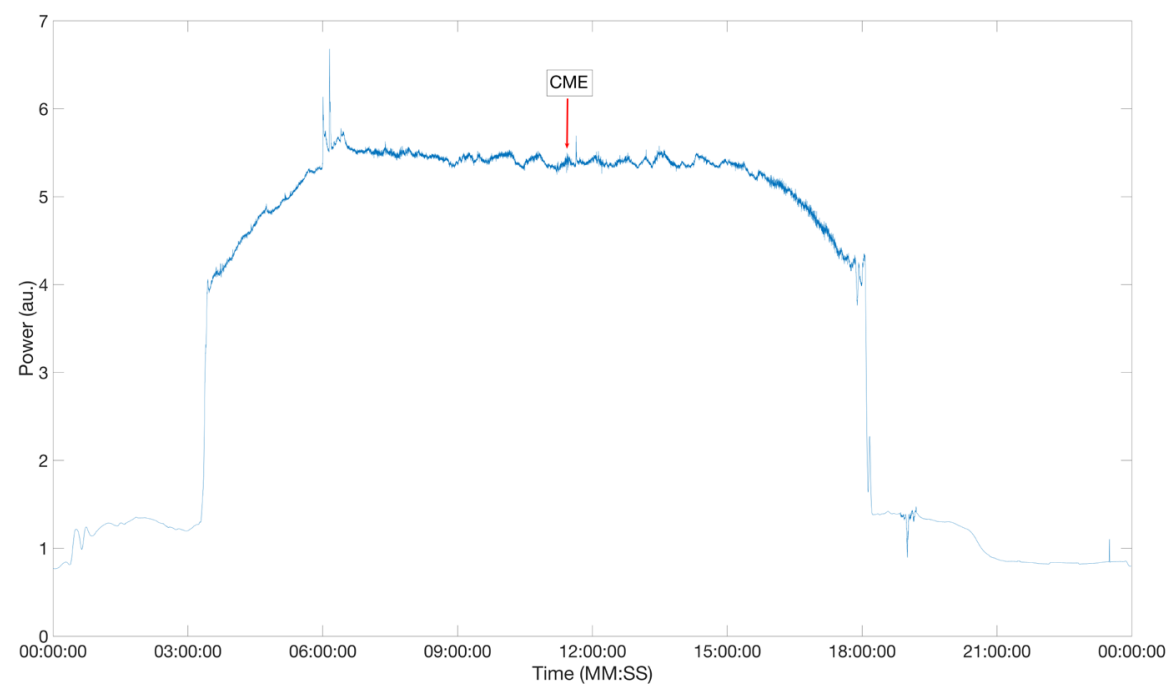

Fig. 4. The solar total intensity at $11.2 \mathrm{GHz}$ on 2 August 2011. CME appearance time is indicated with an arrow. 
Based on high radio frequency (37 $\mathrm{GHz}$ ) observations, the obtained results can be divided into four different categories:

- Category 1 - The maximum radio intensity is achieved after the CME occurs. Totally 8 cases.

- Category 2 - The maximum radio intensity is achieved before the CME occurs. Totally 14 cases.

- Category 3 - The maximum radio intensity is achieved simultaneously when the CME occurs. One case.

- Category 4 - No clear pattern/or not enough observations. One case.

The high frequency $(37 \mathrm{GHz})$ radio brightenings of Category 2 (average $122 \%$ to QSL) were stronger than those of Category 1 (average $114 \%$ to QSL).

In addition, we noticed that CME and 11.2 $\mathrm{GHz}$ radio burst intensity maximum appeared within two to three hours. We could not detect $11.2 \mathrm{GHz}$ burst in every 24 cases. In nine cases of thirteen, $11.2 \mathrm{GHz}$ solar radio burst maximum intensity was detected before CME appeared. It has been reported [11] that CMEs appear between 15 and 30 minutes after flares appear. Most probably $11.2 \mathrm{GHz}$ burst was detected in Category $1(5 / 8)$ than in Category 2 (7/14). The radio intensity at $11.2 \mathrm{GHz}$ was stronger in Category 2 ( $>165 \%$ to QSL) than in Category 1 (120\% to QSL). Some events belonging to Category 2 were so strong that even the receiver signal chain saturated. Therefore, more precise intensity average value cannot be defined.

The intensity of $11.2 \mathrm{GHz}$ bursts was weaker in those events, which appeared after CME (120\% to QSL). The average intensity of those events, which were observed before CME, was > $145 \%$ to QSL. Finally, when we compared millimetre and centimetre wavelength observations to each other, we noticed that centimetre range (2.7 $\mathrm{cm})$ radio burst was detected more probably when radio brightening intensity (at 37 $\mathrm{GHz}$ ) was strong.

\section{CONCLUSIONS}

It has been proposed that CME energies correlate with solar flare appearance. In practice, more intense and energetic CMEs produce solar flares. This scenario also holds for solar radio bursts. Lower frequency $(11.2 \mathrm{GHz})$ radio observations give a reason to speculate that radio burst is a triggering for CME. If a strong radio burst is detected, it is highly possible that CME is following it. Below, a simplified scenario is presented based on our observations.
We will need more observations to confirm our interpretation, especially at lower frequencies. We could utilise, for instance, LOFAR (Low-Frequency Array) observations for this purpose. In addition, more densely sampled intensity profile of radio brightening could give additional information to our scenario. During the next solar cycle, cycle 25 , we will try to observe more these events with a more densely sampled interval. 
This publication makes use of data obtained at Metsähovi Radio Observatory of Aalto University. We acknowl- edge support from the European Union FP7-SPACE-2013-1 programme for the HELCATS project (\#606692).

\section{REFERENCES}

1. Cecatto, J. R. (2009). Observations of Radio Spectra at 1-2.5 Ghz Associated with CME Start Time. Universal Heliophysical Processes, 257, 317-321. doi:10.1017/ S1743921309029482.

2. Aschwanden, M. J. (2004). Physics of the Solar Corona. An Introduction. Berlin: Springer.

3. Isaeva, E. A., \& Tsap, Y. T. (2017). Microwave Emission of Solar Flares: Coronal Mass Ejections and Shock Waves. Odessa Astronomical Publications, 30, 222. doi:10.18524/1810-4215.2017.30.114670.

4. Mondal, S., Oberoi, D., \& Vourlidas, A. (2020). Estimation of the Physical Parameters of a CME at High Coronal Heights Using Low-frequency Radio Observations. The Astrophysical Journal, 893 (1). doi:10.3847/1538-4357/ab7fab.

5. Dididze, G. (2019). Comparative Analysis of Solar Radio Bursts before and during CME Propagation. Astronomy and Astrophysics, 625. doi:10.1051/0004-6361/201629489.

6. Krupar, V. (2019). Statistical Survey of Coronal Mass Ejections and Interplanetary Type II Bursts. The Astrophysical Journal, 882 (2). doi:10.3847/1538-4357/ab3345.
7. Pohjolainen, S., Hildebrandt, J., Karlický, M., Magun, A., \& Chertok, I. M. (2002). Prolonged Millimeter-Wave Radio Emission from a Solar Flare Near the Limb. Astronomy and Astrophysics, 396, 683-692. doi:10.1051/0004-6361:20021431.

8. Kallunki, J., \& Tornikoski, M. (2018). Measurements of the Quiet-Sun Level Brightness Temperature at $8 \mathrm{~mm}$. Solar Physics, 293 (11), article id. 156.

9. Kallunki, J., Tornikoski, M., Tammi, J., Kinnunen, E., Korhonen, K., Kesäläinen, S., \& Arkko, O. (2018). Forty Years of Solar Radio Observations at Metsähovi Radio Observatory. Astronomische Nachrichten, 339 (204), 204-211.

10. Uunila, M., \& Kallunki, J. (2015). Reliability of 1.8-Meter Solar Radio Telescope at Metsähovi Radio Observatory for Longterm Solar Monitoring. Astrophysics and Space Science, 359, article id. 33.

11. Youssef, M. (2013). Statistical Study of the CME-Solar Flares Associated Events. Earth Moon and Planets, 110 (3-4), 185195. doi:10.1007/s11038-013-9419-1. 